\title{
COMPLEX RADIO SPECTRAL ENERGY DISTRIBUTIONS IN LUMINOUS AND ULTRALUMINOUS INFRARED GALAXIES
}

\author{
Adam K. Leroy ${ }^{1,8}$, Aaron S. Evans ${ }^{1,2}$, Emmanuel Momjian ${ }^{3}$, Eric Murphy ${ }^{4}$, Jürgen Ott ${ }^{3}$, Lee Armus $^{5}$, \\ James Condon $^{1}$, Sebastian HaAn ${ }^{5}$, Joseph M. Mazzarella ${ }^{5}$, David S. Meier ${ }^{3,6}$, George C. Privon $^{2}$, \\ Eva SCHINNERER ${ }^{7}$, JASON SURACE $^{5}$, AND FABIAN WALter ${ }^{7}$ \\ ${ }^{1}$ National Radio Astronomy Observatory, 520 Edgemont Road, Charlottesville, VA 22903-2475, USA \\ ${ }^{2}$ Department of Astronomy, University of Virginia, 530 McCormick Road, Charlottesville, VA 22904, USA \\ ${ }^{3}$ National Radio Astronomy Observatory, P.O. Box O, Socorro, NM 87801, USA \\ ${ }^{4}$ Observatories of the Carnegie Institution for Science, 813 Santa Barbara Street, Pasadena, CA 91101, USA \\ ${ }^{5}$ Spitzer Science Center, California Institute of Technology, MC 314-6, Pasadena, CA 91125, USA \\ ${ }^{6}$ New Mexico Institute of Mining and Technology, 801 Leroy Place, Socorro, NM 87801, USA \\ ${ }^{7}$ Max Planck Institut für Astronomie, Königstuhl 17, Heidelberg D-69117, Germany \\ Received 2011 April 15; accepted 2011 June 21; published 2011 August 29
}

\begin{abstract}
We use the Expanded Very Large Array to image radio continuum emission from local luminous and ultraluminous infrared galaxies (LIRGs and ULIRGs) in $1 \mathrm{GHz}$ windows centered at 4.7, 7.2, 29, and $36 \mathrm{GHz}$. This allows us to probe the integrated radio spectral energy distribution (SED) of the most energetic galaxies in the local universe. The 4-8 GHz flux densities agree well with previous measurements. They yield spectral indices $\alpha \approx-0.67$ (where $\left.F_{v} \propto v^{\alpha}\right)$ with $\pm 0.15(1 \sigma)$ scatter, typical of nonthermal (synchrotron) emission from star-forming galaxies. The contrast of our $4-8 \mathrm{GHz}$ data with literature 1.5 and $8.4 \mathrm{GHz}$ flux densities gives further evidence for curvature of the radio SED of U/LIRGs. The SED appears flatter near $\sim 1 \mathrm{GHz}$ than near $\sim 6 \mathrm{GHz}$, suggesting significant optical depth effects at lower frequencies. The high-frequency $(28-37 \mathrm{GHz}$ ) flux densities are low compared to extrapolations from the 4-8 GHz data. We confirm and extend to higher frequency a previously observed deficit of high-frequency radio emission for luminous starburst galaxies.
\end{abstract}

Key words: galaxies: active - galaxies: starburst - infrared: galaxies - radio continuum: galaxies

Online-only material: color figure

\section{INTRODUCTION}

Most very luminous galaxies at $z \sim 0$ emit the bulk of their luminosity in the infrared (IR). These luminous and ultraluminous infrared galaxies (LIRGs and ULIRGs, infrared luminosity $L_{\mathrm{IR}}[8-1000 \mu \mathrm{m}]>10^{11}$ and $\left.L_{\mathrm{IR}}>10^{12} L_{\odot}\right)$ make up the majority of galaxies with $L_{\text {bol }} \gtrsim 10^{11.5} L_{\odot}$. These systems often correspond to major mergers and compared to normal star-forming disk galaxies, U/LIRGs exhibit enhanced, concentrated molecular gas content, high star formation efficiency, increased incidence of active galactic nuclei (AGNs) activity, and disturbed morphologies (Sanders \& Mirabel 1996). Even the closest ULIRGs are $\gtrsim 100 \mathrm{Mpc}$ away with compact radio, IR, and millimeter line emission (e.g., Lonsdale et al. 1993; Downes \& Solomon 1998; Sakamoto et al. 2008), so that high angular resolution, such as that obtained by the Expanded Very Large Array (EVLA), is required to study their structure.

Nonthermal (synchrotron) radio emission is well established to track IR emission (the "radio-FIR correlation"; Helou et al. 1985; Condon 1992; Yun et al. 2001), while thermal (bremsstrahlung) radio emission at $v \gtrsim 4 \mathrm{GHz}$ gives an unobscured view of ionizing photon production. These emission mechanisms, the indifference of radio waves to dust, and the resolving power of $\mathrm{cm}$-wave interferometers make the radio continuum a natural tool to dissect the detailed structure of U/LIRGs. Deviations from the radio-IR correlation, radio morphology, and brightness can all help distinguish the presence and contribution of AGN to the overall luminosity, though not necessarily uniquely (Condon et al. 1991; Lonsdale et al. 1995;

\footnotetext{
8 Hubble Fellow.
}

Sajina et al. 2008; Lonsdale et al. 2006, and references in the latter).

This Letter reports first results from our EVLA program to explore the structure of star formation in U/LIRGs using radio continuum emission. We are imaging 22 of the most luminous nearby U/LIRGs $\left(L_{\mathrm{IR}}>10^{11.6} L_{\odot}\right)$ in the $C(4-8 \mathrm{GHz})$ and $K a(26.5-40 \mathrm{GHz})$ bands. Here we report observations from the EVLA's $C$ and $D$ configurations. The EVLA's continuum sensitivity makes it possible to achieve good signal-to-noise ratio $(\mathrm{S} / \mathrm{N})$, even at high frequencies, with modest time on source. The large instantaneous bandwidth allows us to probe the radio spectral index, $\alpha$ (defined so that $F_{v} \propto v^{\alpha}$ ), both within and between bands.

Here we explore variations in the radio spectral energy distribution (SED) of our targets and in the radio-IR correlation. In a simple picture dominated by star formation we would expect the $C$ band to be dominated by synchrotron emission with spectral index $\alpha \approx-0.8$, while the $K a$ contains an approximately equal mixture of nonthermal emission and thermal emission, which has $v=-0.1$. For systems dominated by an AGN, the spectral index may show wider variation depending on the orientation of the AGN or injection spectrum of electron energies. We close by showing overlays of the $\mathrm{Ka}$-band emission on Hubble Space Telescope (HST) imaging. These highlight the EVLA's improved ability to trace embedded star formation at high resolution.

\section{OBSERVATIONS AND DATA REDUCTION}

Our EVLA resident shared risk observing program, AL746, is imaging radio continuum emission from nearby LIRGs and ULIRGs. The sample, listed in Table 1, consists of 22 of the most 
Table 1

Integrated Properties

\begin{tabular}{|c|c|c|c|c|c|c|c|c|}
\hline Galaxy & $\begin{array}{l}\text { Dist. }^{\mathrm{a}} \\
(\mathrm{Mpc})\end{array}$ & $\begin{array}{c}L_{\mathrm{IR}}^{\mathrm{a}} \\
\left(\log _{10} L_{\odot}\right)\end{array}$ & $\begin{array}{l}S_{5.95^{\mathrm{b}}} \\
(\mathrm{mJy})\end{array}$ & $\alpha_{C}^{\mathrm{c}}$ & $\begin{array}{l}S_{32.5^{b}} \\
(\mathrm{mJy})\end{array}$ & $\begin{array}{c}S_{1.49}(\mathrm{C} 91)^{\mathrm{d}} \\
(\mathrm{mJy})\end{array}$ & $\begin{array}{c}S_{8.44}(\mathrm{C} 91)^{\mathrm{d}} \\
(\mathrm{mJy})\end{array}$ & $\begin{array}{c}S_{15}(\mathrm{C} 08)^{\mathrm{d}} \\
\quad(\mathrm{mJy})\end{array}$ \\
\hline CGCG 436-030 & 134 & 11.69 & $18.6 \pm 0.04$ & -0.72 & $8.6 \pm 0.6$ & 43.1 & 12.7 & 8.73 \\
\hline CGCG 448-020 (II Zw 096) & 161 & 11.94 & $14.6 \pm 0.06$ & -0.66 & $6.8 \pm 1.1$ & $\ldots$ & $\ldots$ & $\ldots$ \\
\hline III Zw 035 & 119 & 11.64 & $25.4 \pm 0.05$ & -0.50 & $7.3 \pm 0.6$ & 39.3 & 19.7 & 9.7 \\
\hline IRAS $19542+1110$ & 295 & 12.12 & $9.5 \pm 0.04$ & -0.79 & $3.4 \pm 0.7$ & $\ldots$ & $\ldots$ & $\ldots$ \\
\hline IRAS $21101+5810$ & 174 & 11.81 & $9.8 \pm 0.04$ & -0.67 & $4.6 \pm 0.6$ & $\ldots$ & $\ldots$ & $\ldots$ \\
\hline IRAS F01365-1042 & 210 & 11.85 & $10.0 \pm 0.04$ & -0.47 & $4.1 \pm 0.5$ & 17.0 & 8.2 & 3.97 \\
\hline IRAS $0857+39$ & 264 & 12.16 & $4.44 \pm 0.04$ & -0.29 & $\ldots{ }^{\mathrm{e}}$ & 6.5 & 4.1 & 3.18 \\
\hline IRAS $1525+36$ & 254 & 12.08 & $12.0 \pm 0.04$ & -0.44 & $4.3 \pm 0.8$ & 12.8 & 10.5 & 4.2 \\
\hline IRAS $1713+53$ & 232 & 11.96 & $9.3 \pm 0.04$ & -0.78 & $4.1 \pm 0.7$ & 28.4 & 8.9 & $\ldots$ \\
\hline IRAS F23365+3604 & 287 & 12.20 & $10.6 \pm 0.04$ & -0.72 & $2.4 \pm 0.5$ & $\ldots$ & $\ldots$ & $\ldots$ \\
\hline MCG +07-23-019 (Arp 148) & 158 & 11.62 & $16.0 \pm 0.06$ & -0.66 & $5.1 \pm 1.2$ & $\ldots$ & $\ldots$ & $\ldots$ \\
\hline NGC 3690 (Mrk 171) & 51 & 11.93 & $275.5 \pm 0.34$ & -0.62 & $89.0 \pm 2.6$ & 658.0 & $\ldots$ & $\ldots$ \\
\hline UGC 04881 (Arp 55) & 178 & 11.74 & $11.4 \pm 0.09$ & -0.96 & $3.1 \pm 1.0$ & 29.0 & 8.8 & 3.5 \\
\hline UGC 05101 & 177 & 12.01 & $61.5 \pm 0.08$ & -0.75 & $16.8 \pm 1.1$ & 146.0 & 52.6 & 18.0 \\
\hline UGC 08058 (Mrk 231) & 192 & 12.57 & $312.8 \pm 0.2$ & -0.17 & $88 \pm 3.5$ & 240.0 & 265.0 & $\ldots$ \\
\hline UGC 08387 (Arp 193, I Zw 056) & 110 & 11.73 & $46.3 \pm 0.08$ & -0.55 & $16.5 \pm 0.9$ & 106.0 & 34.9 & 7.5 \\
\hline UGC 08696 (Mrk 273, I Zw 071) & 173 & 12.21 & $60.3 \pm 0.08$ & -0.72 & $19.5 \pm 1.0$ & 130.0 & 43.5 & 14.3 \\
\hline UGC 09913 (Arp 220) & 88 & 12.28 & $194.5 \pm 0.08$ & -0.59 & $59.5 \pm 2.5$ & 301.1 & 148.0 & $\ldots$ \\
\hline VII Zw 031 & 240 & 11.99 & $12.5 \pm 0.04$ & -0.91 & $3.7 \pm 0.7$ & $\ldots$ & $\ldots$ & $\ldots$ \\
\hline VV 250a & 142 & 11.81 & $19.6 \pm 0.05$ & -0.69 & $3.8 \pm 1.2$ & 51.2 & 17.0 & $\cdots$ \\
\hline VV 340a & 157 & 11.74 & $23.7 \pm 0.12$ & -1.06 & $\ldots{ }^{\mathrm{e}}$ & 68.8 & $\ldots$ & $\ldots$ \\
\hline VV 705 & 183 & 11.92 & $19.6 \pm 0.05$ & -0.75 & $4.8 \pm 0.8$ & 46.8 & 12.1 & 3.6 \\
\hline
\end{tabular}

Notes.

${ }^{a}$ Hubble flow distance and IR luminosity adopted from Sanders et al. (2003) following Armus et al. (2009).

${ }^{\mathrm{b}}$ Flux density integrated over significant $C$-band emission. See the text. Uncertainties are only statistical, bootstrapped from the map.

${ }^{c}$ Intensity-weighted in-band spectral index derived from MFS imaging. See the text.

d Literature flux densities from Condon et al. (1991) and Clemens et al. (2008).

e $\mathrm{S} / \mathrm{N}<3$ integrated over the $C$-band beam. Still detected at $\mathrm{Ka}$ resolution.

luminous members of the IRAS Revised Bright Galaxy Sample (RBGS; Sanders et al. 2003) northern enough to be observed by the EVLA. Distances span 50-300 Mpc, with a median of $177 \mathrm{Mpc}$, and IR luminosities go from $\log _{10} L_{\mathrm{IR}}\left[L_{\odot}\right]=$ 11.6-12.6.

The whole program consists of imaging each target at four frequencies, $v \sim 4.7,7.2,29$, and $36 \mathrm{GHz}$, with a bandwidth of $1 \mathrm{GHz}$ centered at each frequency. We observe the two low-frequency windows (together the " $C$-band" data) and two high frequencies (the " $K a$-band" data) simultaneously. Here we report the results from the two most compact EVLA configurations $(C$ and $D$ ). We observed each target for $\sim 5$ minutes in each band and configuration.

We reduced the data using the Common Astronomy Software Applications (CASA) package developed by NRAO. We adopted standard approaches to flag bad data (radio frequency interference is a particular issue in the $C$ band) and calibrate the bandpass, phase, and amplitude response of individual antennas. We iteratively self-calibrated the data whenever possible.

We combined all data for each band into a single image using CASA's multi-frequency synthesis (MFS) capabilities (see Sault \& Wieringa 1994). This image reconstruction mode synthesizes interferometer observations across a wide bandwidth. It accounts for changes in the synthesized beam as a function of frequency and produces images of intensity for a fiducial frequency and maps of the spectral index. For our data, these

\footnotetext{
9 This refers to estimates of the "total" $(8-1000 \mu \mathrm{m})$ IR luminosity constructed from IRAS flux densities by Sanders et al. (2003) following Sanders \& Mirabel (1996).
}

fiducial frequencies are $5.95 \mathrm{GHz}$ for the $C$ band and $32.5 \mathrm{GHz}$ for the $K a$ band. Note that we do not actually observe at either of these frequencies, they are merely an intermediate between our two separated $1 \mathrm{GHz}$ windows. At $C$ band, we deconvolved (CLEAN) the image using components that have both a flux density and a spectral index. At $K a$ band we use components that have only flux density at the fiducial frequency because we expect a flatter spectral index, have a lower $\mathrm{S} / \mathrm{N}$, and have a smaller fractional bandwidth.

After reduction, we have maps of intensity for $\mathrm{C}$ and $\mathrm{Ka}$ bands and spectral index at the $C$ band. Synthesized beams are $\sim 8^{\prime \prime}$ at the $C$ band and $\sim 1^{\prime \prime}$ at the $K a$ band. The noise in a $C$-band image is usually $\approx 20 \mu \mathrm{Jy}$ beam $^{-1}$, though for our brightest targets calibration issues sometimes limit us to about twice this value. A typical noise in a $K a$-band image is $\approx 50 \mu \mathrm{Jy}_{\text {beam }}{ }^{-1}$. All targets are detected, with peak $\mathrm{S} / \mathrm{N}$ from 200 to $6.3 \times 10^{3}$ at the $C$ band (median 670 ) and 12 to 500 at the $K a$ band (median 50).

We measure $C$-band flux densities in the following way: we estimate the rms noise away from the source. We then identify the region of contiguous $\mathrm{S} / \mathrm{N}>10$ emission that contains the galaxy. We integrate over this area to derive the flux density. We take the intensity-weighted mean of the spectral index $\left\langle\alpha_{\mathrm{C}}\right\rangle=\int F_{\nu} \alpha / \int F_{\nu}$ derived by the MFS CLEAN as the spectral index for the source. We derive $K a$-band flux densities by integrating over the same area used to derive the $C$-band flux densities. Over this matched area, two galaxies have integrated $\mathrm{S} / \mathrm{N}<3$ at $\mathrm{Ka}$ and we omit them from all $\mathrm{Ka}$-band related analysis. 

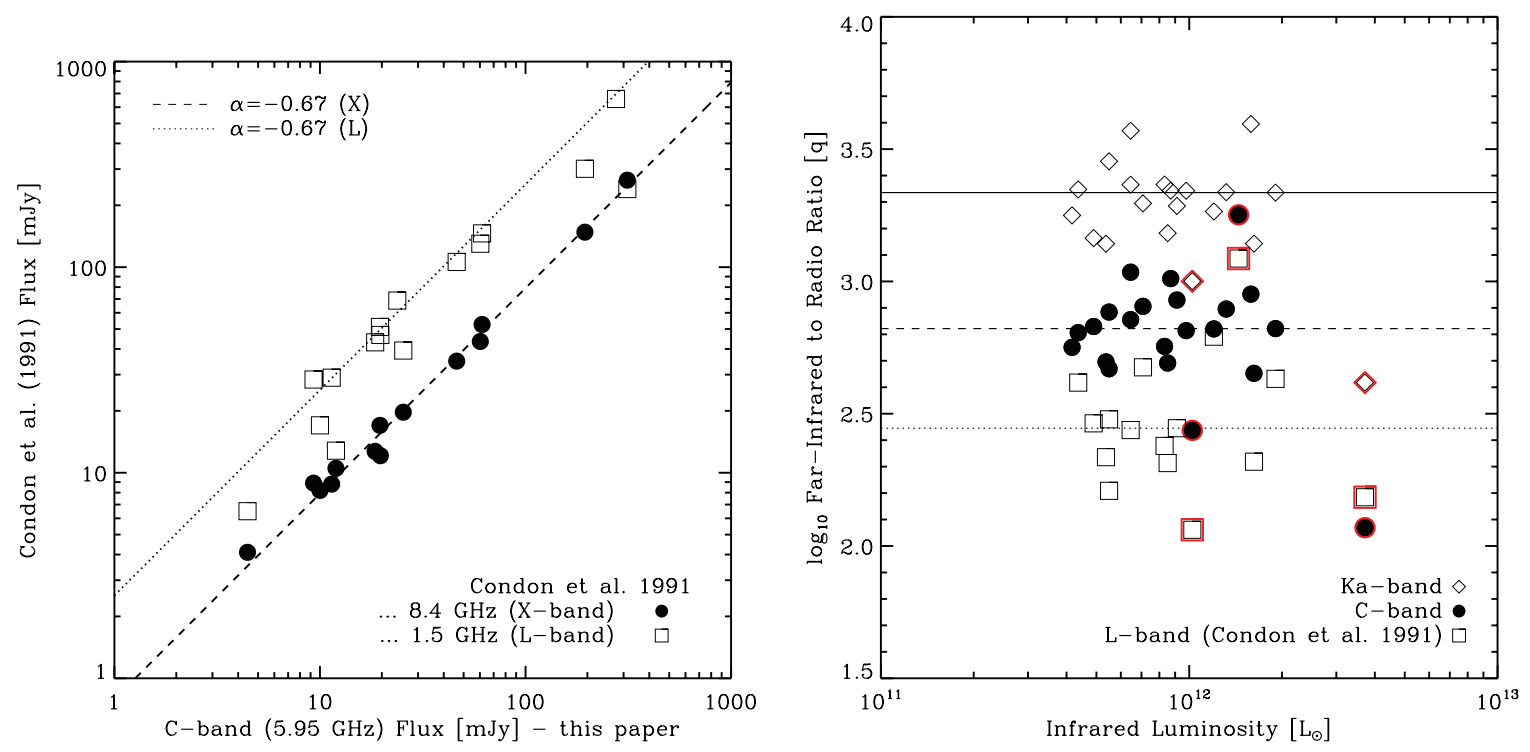

Figure 1. Left: flux densities from Condon et al. (1991) ( $y$-axis) as a function of our $5.95 \mathrm{GHz}(C$-band) flux density ( $x$-axis). Filled circles show $X$-band $(8.4 \mathrm{GHz})$ flux densities. Open squares show $L$-band $(1.5 \mathrm{GHz})$ flux densities. Dashed lines show the expected flux density given our $C$-band flux density and a spectral index $\alpha=-0.67$. Our $C$-band flux densities agree well with the $X$-band values. The $L$-band flux densities tend to lie below the expected value, indicating curvature in the radio SED in the range 1-5 GHz. Right: ratio of far-infrared to radio emission $(q)$ for $K a$ (open diamonds), $C$ (black circles), and $L$ (open squares) bands. All three bands correlate with IR emission, as expected, with the lowest scatter in the $C$ band. The highest luminosity source, with an inverted spectrum, is UGC 08058 (Markarian 231), which is dominated by an AGN. Red outlines highlight source and two other outliers discussed in the text.

(A color version of this figure is available in the online journal.)

\section{RESULTS}

\subsection{A Typical Synchrotron Spectrum at the C Band}

The left panel of Figure 1 compares our measured $C$-band flux densities at $5.95 \mathrm{GHz}$ ( $x$-axis) to previous measurements at 8.4 GHz (black circles) and $1.5 \mathrm{GHz}$ (open squares) both drawn from Condon et al. (1991). Table 1 reports internal $C$-band spectral indices, $\alpha_{\mathrm{C}}$. Our median $\alpha_{\mathrm{C}}$ is -0.67 with $1 \sigma$ scatter $\approx 0.15$. The $\mathrm{S} / \mathrm{Ns}$ of the data are very high but an uncertainty of even a few percent in the relative calibrations of the two parts of the $C$ band (e.g., due to calibration problems, radio frequency interference, uncertainties in the primary calibrator model, or CLEAN issues) will lead to uncertainties $\sim 0.1$.

We expect nonthermal (synchrotron) emission to dominate the $C$ band. Our measured $\alpha=-0.67$ is close to a normal synchrotron spectral index for a star-forming galaxy, $\alpha \approx-0.8$ (Condon 1992) though AGN can produce similar indices. For $\alpha_{\mathrm{C}}=-0.67$, Figure 1 shows that our $C$-band flux densities agree with the $X$-band measurements of Condon et al. (1991) well. The median ratio after correcting to the same band using $\alpha_{\mathrm{C}}=-0.67$ is $\approx 1.02$ and the scatter is $14 \%$, indicating very good agreement. Thus, our $C$-band measurements from $\approx 4.2-5.2$ and $6.7-7.7 \mathrm{GHz}$ and previous measurements at $8.44 \mathrm{GHz}$ all exhibit good consistency with a typical synchrotron spectrum.

\subsection{Departures From a Simple SED at Low and High v}

In the simple two-component model outlined in the introduction, synchrotron emission with $\alpha \sim-0.8$ dominates below $v \sim 10 \mathrm{GHz}$. Above this frequency, the radio continuum results from a mixture of synchrotron and thermal emission, which has $\alpha=-0.1$. In a normal star-forming galaxy, the ratio of these two processes may be approximately fixed. Figure 2 shows the radio SEDs of our targets, which exhibit significant departures from this picture at both high and low frequencies.
We normalize each SED at $5.95 \mathrm{GHz}$, show literature flux densities from Condon et al. (1991) and Clemens et al. (2008), and overplot the simple two-component, optically thin model from Condon (1992). The lines through our $C$-band points show our measured $\alpha_{\mathrm{C}}$.

At low frequencies, the $1.5 \mathrm{GHz}$ ( $L$ band) flux densities lie mostly below an extrapolation of the synchrotron emission measured in the $C$ band, which appears as a dashed line in Figure 2. This can be seen from the left panel in Figure 1 where the $1.5 \mathrm{GHz}$ points (open squares) tend to scatter below the value expected for our measured $\alpha_{\mathrm{C}}$ (dashed). Our $C$-band flux densities and $\alpha_{\mathrm{C}}$ demonstrate normal synchrotron emission at $v \gtrsim 4 \mathrm{GHz}$. The low $L$-band flux densities imply that the radio SED becomes flatter ( $\alpha$ approaches 0 ) near $v \sim 1 \mathrm{GHz}$. This effect was previously inferred from unusually flat (near 0 ) spectral indices contrasting $1.5-8.4 \mathrm{GHz}$ (Condon et al. 1991) and directly observed via long wavelength observations by Clemens et al. (2010).

For a normal star-forming galaxy, we expect the radio SED to contain an increasing fraction of thermal emission $(\alpha=-0.1)$ moving toward higher frequencies. By $\sim 30 \mathrm{GHz}$ (the $\mathrm{Ka}$ band), the SED of such a galaxy would contain roughly equal parts thermal and nonthermal emission with flatter $\alpha$ than at $5.95 \mathrm{GHz}$. Figure 2 shows that this simple expectation does not hold for our data. Our $\mathrm{Ka}$-band flux densities tend to lie below the simple model: the model predicts a typical $C$-to- $K a$ ratio $\approx 2.4$, while our median ratio is $\approx 3.15$ with $1 \sigma$ scatter 0.75 . The radio spectrum is significantly steeper than expected moving to higher frequencies.

A steep SED at high frequencies has been previously observed in U/LIRGs by Clemens et al. (2008), and the dwarf starburst NGC 1569 (Lisenfeld et al. 2004). If there is a significant thermal contribution at $v \sim 30 \mathrm{GHz}$, and these must be based on the high level of star formation in these systems, then the synchrotron spectral index must be steepening. Clemens et al. (2008) discuss possible mechanisms for this and Clemens et al. 


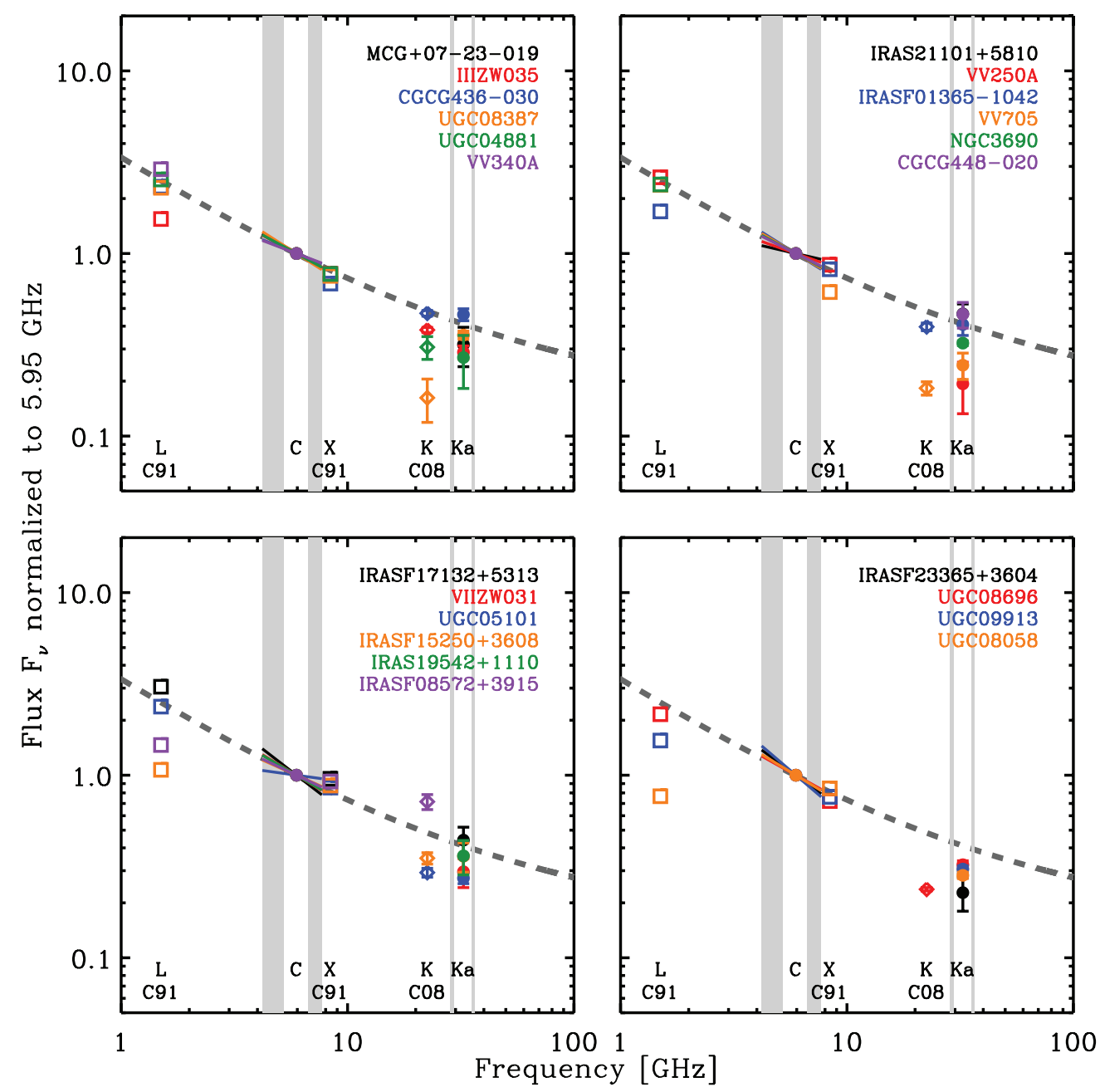

Figure 2. Normalized SEDs of our targets, sorted by IR luminosity from top left to bottom right. Gray regions show our observed frequencies and circles indicate our measured flux densities. Lines show $\alpha_{\mathrm{C}}$ across our $C$-band measurement. We normalize all values to the $5.95 \mathrm{GHz}$ value and plot literature measurements as open symbols. The dashed line shows a simple two-component model mixing optically thin synchrotron $(\alpha=-0.8)$ with thermal emission $(\alpha=-0.1)$ at levels appropriate for a star-forming galaxy (Condon 1992). Our $C$-band measurements show an approximately normal synchrotron shape in most sources, but U/LIRGs depart from this simple model at low and high frequencies. Our new $K a$ measurements confirm a systematic deficit of high-frequency emission, extending this result to higher frequencies $(v \sim 36 \mathrm{GHz})$.

(2010) revisit the topic, while Lisenfeld et al. (2004) discuss possible explanations for a similar observation in the starburst NGC 1569. Allowing that the thermal emission should be present, the synchrotron emission must be depressed at high frequency. Depressed synchrotron emission could result from synchrotron and inverse Compton losses in aging starbursts or regions with high magnetic fields. However, there is no evidence that we observe all of these galaxies at a preferential time and these explanations appear inadequate to match the magnitude of the effect. Alternatively, the injection spectrum of electron energies could differ from those in normal galaxies. This is possible for an AGN, but less plausible for radio continuum emission originating from star formation. We suspect that a modified injection spectrum leads to a deficit of high-frequency synchrotron emission and drives the high-frequency flux deficit. This is a topic that we will investigate further with our full highresolution data.

Depressed synchrotron at high frequencies may be a way to distinguish systems where the radio is dominated by AGNs. We expect a minimum level of thermal emission in systems with radio emission due mostly to star formation, so AGNs likely provide most of the radio emission in the steepest spectrum sources.

\subsection{Radio-Infrared Correlation}

Despite the surprises in the radio SED, the radio and infrared fluxes still track one another well. We calculate the far-infrared-to-radio ratio defined by Helou et al. (1985) as $q_{v}=\log _{10}\left(F_{\mathrm{FIR}} / S_{v}\right)$ with $F_{\mathrm{FIR}}$ the far-IR flux ${ }^{10}$ in units of $3.75 \times 10^{12} \mathrm{~W} \mathrm{~m}^{-2}$ and $S_{v}$ in $\mathrm{W} \mathrm{m}^{-2} \mathrm{~Hz}^{-1}$. The median $q_{5.95}=2.8$ with a scatter of $\approx 0.16$ dex. The $K a$ band shows comparable scatter. The $L$-band $q_{1.5}$ has $\sim 50 \%$ more scatter, $\sim 0.25$ dex. Yun et al. (2001) observed 0.26 dex scatter in the $L$-band radio-IR correlation and 0.33 dex scatter for LIRGs and ULIRGs. The smaller overall scatter in our sample may simply be a result of small number statistics. We interpret the lower scatter at high frequencies in our sample as a manifestation of the same phenomenon that leads to higher scatter in $q_{1.5}$ at high luminosities. The curved SED at low frequencies in luminous IR galaxies adds scatter to the radio-IR correlation as physical processes other than just star formation become important. At higher frequencies and in lower luminosity galaxies the effects are less important. 10 To calculate $q$ we use the IRAS-defined far-IR flux, which captures flux
from 40 to $120 \mu \mathrm{m}$, not the integrated IR flux quoted elsewhere in this Letter. 

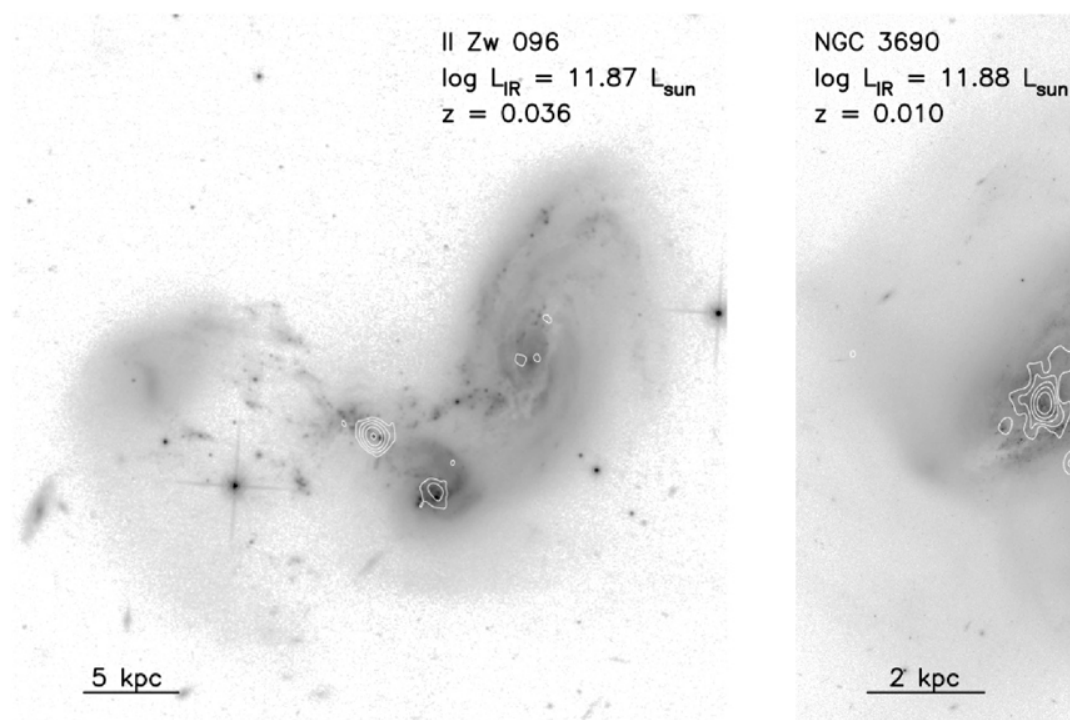

Figure 3. EVLA Ka-band contours superimposed on gray-scale HST ACS/WFC I-band (F814W) images of CGCG 448-020 (II Zw 096, left) and NGC 3690 (right), both on a logarithmic stretch. The contours are displayed in six evenly spaced log steps between $10 \%-100 \%$ of the peak intensity for II $\mathrm{Zw} 096$ and $2 \%-100 \%$ of the peak intensity for NGC 3690 .

The correlation among the radio bands is stronger than the correlation with the IR, with the strongest correlation among the two synchrotron dominated bands: $q_{5.95}$ and $q_{32.5}$ rank correlate with $0.67 ; q_{5.95}$ and $q_{1.5}$ exhibit a rank correlation coefficient of $0.81 ; q_{1.5}$ and $q_{32.5}$ have a rank correlation of 0.57 .

Three galaxies lie notably off the radio-IR correlation. The radio emission from UGC 08058 (Markarian 231) is much $(4.5 \sigma)$ brighter than one would derive from the IR emission. UGC 05101 shows a weaker $(2.5 \sigma)$ excess in the same direction. Both galaxies show evidence for AGN in VLBI observations (Lonsdale et al. 1993, 2003). Though the contribution of the AGN to the bolometric luminosity is debated, Veilleux et al. (2009) estimate the fraction of total IR emission due to the AGN to be $\sim 70 \% \pm 15 \%$ in UGC 08058 and $\sim 35 \% \pm 15 \%$ in UGC 05101 (see Armus et al. 2007 for an even lower estimate).

IRAS F08572+3915 shows unusually $(2.5 \sigma)$ strong IR emission. This is our faintest target and its IR-excess has been noted before (see discussion in Yun et al. 2004) with $~ 25 \%$ of the IR emission in this galaxy associated with a very hot $T \gtrsim 300 \mathrm{~K}$ dust component (Armus et al. 2007, the largest such fraction in their sample). The flat spectral index at low frequencies and the high IR-to-radio ratio in this galaxy could be interpreted to indicate a very young starburst (e.g., Roussel et al. 2006). For example, this galaxy sits next to the youngest age model point in the diagrams of Bressan et al. (2002). However, based on near- and mid-IR spectroscopy (Armus et al. 2007; Risaliti et al. 2010) and X-ray hardness (Iwasawa et al. 2011) much of the luminosity is thought to come from a deeply embedded AGN in the northwest nucleus. In either case, a luminous, compact, deeply embedded object drives the luminosity and results unusually strong IR emission. We detect it at $K a$ band, but not at $>3 \sigma$ over the $C$-band aperture so that further study will have to wait for the better $\mathrm{S} / \mathrm{N}$ and smaller matched apertures from our full data set.

\subsection{Tracing Embedded Star Formation}

Figure 3 shows our $\mathrm{Ka}$-band emission superimposed on I-band HST images for two targets, CGCG 448-020 (II Zw 096, left) and NGC 3690 (right). The images show the utility of combining high-resolution radio data with the multiwavelength data assembled by the Great Observatory All-sky LIRG Survey (GOALS; Armus et al. 2009) to assess the distribution and strength of embedded star formation and AGN activity. In II $\mathrm{Zw}$ 096, the brightest radio continuum point does not have an obvious optical counterpart. This bright radio emission coincides with the peak of the IR emission measured by Spitzer (Inami et al. 2010), underscoring that this is a star-forming peak without an obvious optical signature. In NGC 3960 (right), several of the radio sources have optical counterparts, but without the radio emission it is difficult to determine which optical knots harbor luminous embedded activity. Here too, the $32.5 \mathrm{GHz}$ continuum peaks coincide with the bright IR emission (Alonso-Herrero et al. 2010) but with higher angular resolution.

\subsection{Summary}

We present new measurements of radio continuum emission from $22 \mathrm{U} / \mathrm{LIRG}$ across $1 \mathrm{GHz}$ windows centered at 4.7, 7.2, 29, and $36 \mathrm{GHz}$. Our observations are consistent with typical synchrotron emission at 4.2-7.7 GHz. Comparing these normal $C$-band results with new high-frequency $29-36 \mathrm{GHz}$ fluxes and literature measurements at $L, X$, and $K$ bands, we see that the radio SEDs of U/LIRGs systematically deviate from a simple two-component model at both low and high frequencies. The new EVLA data here provide (1) observations filling in previously unsampled parts of the radio SED; (2) a clear observation of "normal" synchrotron behavior in the range $4-8 \mathrm{GHz}$, reinforcing that the radio SED must be curving away from simple expectations; and (3) an observation of systematically low highfrequency flux density at higher frequencies than have been previously studied. The radio fluxes correlate with IR emission as expected, with some suggestion that the $C$ and $K a$ bands exhibit a more direct link to IR emission than the $L$ band.

An ongoing extension of this project to all EVLA configurations will dramatically improve our resolution and $\mathrm{S} / \mathrm{N}$ in both frequency bands. Combining these data with the multiwavelength data available for our targets, we expect to test if the star formation surface density exhibits any maximum value (e.g., the limit set by momentum-driven winds suggested by 
Murray et al. 2005), derive characteristic sizes for the starforming disks, map the spectral index at $\lesssim 0^{\prime \prime} .5$ resolution, and explore the implications of the brightness temperature and radio SED for the dominant radio emission mechanism.

We thank the anonymous referee for suggestions that improved this work. The National Radio Astronomy Observatory is a facility of the National Science Foundation operated under cooperative agreement by Associated Universities, Inc. Support for A.K.L. during part of this work was provided by NASA through Hubble Fellowship grant HST-HF-51258.01-A awarded by the Space Telescope Science Institute, which is operated by the Association of Universities for Research in Astronomy, Inc., for NASA, under contract NAS 5-26555.

\section{REFERENCES}

Alonso-Herrero, A., Pereira-Santaella, M., Rieke, G. H., et al. 2010, Adv. Space Res., 45, 99

Armus, L., Charmandaris, V., Bernard-Salas, J., et al. 2007, ApJ, 656, 148

Armus, L., Mazzarella, J. M., Evans, A. S., et al. 2009, PASP, 121, 559

Bressan, A., Silva, L., \& Granato, G. L. 2002, A\&A, 392, 377

Clemens, M. S., Scaife, A., Vega, O., \& Bressan, A. 2010, MNRAS, 405, 887
Clemens, M. S., Vega, O., Bressan, A., et al. 2008, A\&A, 477, 95

Condon, J. J. 1992, ARA\&A, 30, 575

Condon, J. J., Huang, Z., Yin, Q. F., \& Thuan, T. X. 1991, ApJ, 378, 65

Downes, D., \& Solomon, P. M. 1998, ApJ, 507, 615

Helou, G., Soifer, B. T., \& Rowan-Robinson, M. 1985, ApJ, 298, L7

Inami, H., Armus, L., Surace, J. A., et al. 2010, AJ, 140, 63

Iwasawa, K., Sanders, D. B., Teng, S. H., et al. 2011, A\&A, 529, A106

Lisenfeld, U., Wilding, T. W., Pooley, G. G., \& Alexander, P. 2004, MNRAS, 349,1335

Lonsdale, C. J., Farrah, D., \& Smith, H. E. 2006, in Ultraluminous Infrared Galaxies, ed. J. W. Mason (Berlin: Springer), 285

Lonsdale, C. J., Lonsdale, C. J., Smith, H. E., \& Diamond, P. J. 2003, ApJ, 592 , 804

Lonsdale, C. J., Smith, H. E., \& Lonsdale, C. J. 1995, ApJ, 438, 632

Lonsdale, C. J., Smith, H. J., \& Lonsdale, C. J. 1993, ApJ, 405, L9

Murray, N., Quataert, E., \& Thompson, T. A. 2005, ApJ, 618, 569

Risaliti, G., Imanishi, M., \& Sani, E. 2010, MNRAS, 401, 197

Roussel, H., Helou, G., Smith, J. D., et al. 2006, ApJ, 646, 841

Sajina, A., Yan, L., Lutz, D., et al. 2008, ApJ, 683, 659

Sakamoto, K., Wang, J., Wiedner, M. C., et al. 2008, ApJ, 684, 957

Sanders, D. B., Mazzarella, J. M., Kim, D., Surace, J. A., \& Soifer, B. T. 2003, AJ, 126, 1607

Sanders, D. B., \& Mirabel, I. F. 1996, ARA\&A, 34, 749

Sault, R. J., \& Wieringa, M. H. 1994, A\&AS, 108, 585

Veilleux, S., Rupke, D. S. N., Kim, D.-C., et al. 2009, ApJS, 182, 628

Yun, M. S., Reddy, N. A., \& Condon, J. J. 2001, ApJ, 554, 803

Yun, M. S., Reddy, N. A., Scoville, N. Z., et al. 2004, ApJ, 601, 723 